\title{
Constipation and other chronic gastrointestinal problems in spinal cord injury patients
}

\author{
D De Looze ${ }^{1}$, M Van Laere ${ }^{2}$, M De Muynck ${ }^{3}, \mathrm{R}_{\text {Beke }}^{4}$ and A Elewaut ${ }^{5}$ \\ ${ }^{1}$ Gastroenterologist; ${ }^{2}$ Professor of Physical Medicine and Rehabilitation, Head of Rehabilitation Centre; ${ }^{3}$ Consultant \\ in Physical Medicine and Rehabilitation; ${ }^{4}$ Pharmacist; ${ }^{5}$ Professor of Gastroenterology, Ghent University Hospital, De \\ Pintelaan 185, B-900 Gent, Belgium
}

\begin{abstract}
From a questionnaire sent to 90 spinal cord injury (SCI) patients it is concluded that $58 \%$ of patients with a complete SCI above L2 suffer from constipation, defined as two or fewer bowel movements per week, or the use of aids such as laxatives, manual evacuation or enemas. Tetraplegic patients had the highest prevalence of constipation, while patients with low paraplegia were less prone to constipation. The use of anticholinergic drugs was found to predispose to constipation. Preserved rectal sensation did not influence the presence of constipation. Faecal incontinence was rare. Regular abdominal pain was present in one third of SCI patients and might be caused by an irritable bowel syndrome in $62 \%$ of these.
\end{abstract}

Keywords: SCI; constipation; faecal incontinence; abdominal pain

\section{Introduction}

Adequate control of potentially life-threatening complications after spinal cord injury (SCI), such as pulmonary and urinary infections, has generated more interest in the quality of life of these patients. Urogenital dysfunction after a traumatic cord lesion has been extensively studied but literature data on the prevalence of gastrointestinal disorders are scarce although these also represent a distressing problem for SCI patients. ${ }^{1,2}$ The term 'traumatic constipation' was introduced by Devroede et al for patients who had developed constipation after lumbar spinal trauma. ${ }^{3} \mathrm{~A}$ large-scale study based on ICD (International Classification of Diseases) coding has shown a strong association between constipation and neurological diseases including SCI. ${ }^{4}$ Abdominal pathology following damage to the central nervous system may present in an atypical way because of the disturbed somatic and visceral sensation, the change in intestinal motility and the loss of voluntary control of the anal sphincter. Problems with defaecation, varying from faecal incontinence to chronic constipation, may have severe implications for the patient's reintegration into society. Many SCI patients are on high-fibre diets and laxatives, or spend much time in emptying their bowel. On the other hand, bowel movements can occur unexpectedly, which may frighten some patients and isolate them eg from sports and social activities. Faecal impaction can also present as a medical emergency if life-threatening autonomic dysreflexia occurs. ${ }^{5}$

The present study was designed to analyse the prevalence of constipation in patients with SCI and to

Correspondence: D De Looze look for predisposing factors. Associated phenomena such as faecal incontinence and abdominal pain were also evaluated. To this end a questionnaire was sent to 90 patients who had a complete spinal cord lesion.

\section{Subjects and methods}

\section{Subjects}

In the last 4 months of 1994 a questionnaire was sent to 90 patients with a complete spinal cord lesion. They were randomly chosen from a population that had previously been treated at the Ghent Rehabilitation Centre. They all had a complete neurological lesion for more than 5 months. The clinical-neurological level was determined according to the guidelines of the American Spinal Injury Association. ${ }^{6}$ Patients admitted to the rehabilitation centre were not included.

Correctly completed replies were obtained from 78 patients, i.e. a response rate of $87 \%$. This study population consisted of 63 men and 15 women with a median age of 37.5 years (range $18-72$ years). The median duration of the spinal cord lesion at the time of the analysis was 7 years (range 5 months -22 years). The cause was traumatic in 71 and medical in seven (spinal cord bleeding, medullary infarction following a coagulation disorder, tumor resection, scoliosis correction, disk herniation, Pott's disease and Kahler's disease).

The neurological level of the injury was the most caudal level which was tested intact for motor function. There were 23 tetraplegic and 55 paraplegic patients (32 with lesions above T10, 23 T10 and lower). The lowest level of injury was L2. 


\section{Methods}

Table 1 shows the items that were subject to extensive multiple choice questions. We used modified Rome criteria for the definition of constipation: "two or less bowel movements a week, or the use of laxatives or enema more than once a week, or digital evacuation of faeces on all occasions. ${ }^{7}$ Patients who fulfilled these criteria were referred to as the constipation group (group C); patients with normal stool habits according to our criteria were designated as the non-constipation group (group NC). The use of anal stimulation in order to precipitate rectal evacuation was not considered a manifestation of constipation.

For the evaluation of abdominal pain, we applied the Rome-criteria for Irritable Bowel Syndrome (IBS) to our patient group. Irritable Bowel Syndrome was defined as: 'abdominal pain which is relieved by defaecation and two or more of the following conditions: altered stool frequency, altered stool consistency, altered stool passage, passage of mucus and feeling of abdominal distension.

\section{Statistical analysis}

The $\chi^{2}$-method was used to analyse differences between the patient groups. The Mann-Whitney U-test was used to compare ages and duration of the lesions between the patient groups. Significance was accepted if $P<0.05$.

\section{Results}

Prevalence of constipation

Forty-five $(58 \%)$ patients belonged to group C. The remaining 33 patients belonged to group NC. Twentythree patients had a bowel frequency of two or less per week, 15 patients needed manual evacuation of faeces on all occasions and 15 patients used laxatives on a regular basis. Anal stimulation was regularly used by $64(82 \%)$ patients.

Table 1 Questionnaire items

\begin{tabular}{l} 
Personal data \\
Age, Sex \\
Neurological data \\
Cause, date and level of injury \\
Medical history prior to injury \\
e.g. anticholinergics, muscle relaxants, laxatives \\
Dietary adjustment \\
Bowel habit \\
Frequency \\
Use of aids, e.g. manual evacuation, anal stretch, \\
suppositories \\
Consistency \\
Abdominal pain \\
Abdominal distension \\
Faecal continence \\
Sensation: urge to defaecate, rectal sensation \\
\hline
\end{tabular}

\section{Patient characteristics}

Both patient groups were similar as far as age, sex and duration of the injury were concerned. Group $\mathrm{C}$ contained 38 men and seven women with a median age of 32 years $(18-72)$. Group NC comprised 25 men and eight women with a median age of 32 years (1959). The median duration of SCI was 57 months (5$249)$ in group $\mathrm{C}$ and 77 months $(7-268)$ in group NC. Medical history prior to the SCI was similar in both groups (two patients had had an appendectomy).

\section{Level of cord injury}

Figure 1 depicts the presence of constipation according to the level of cord injury. A difference was found between those with tetraplegia, paraplegia above T10 and paraplegia below T10. Constipation seemed to be more frequent in those who were tetraplegic but this did not reach statistical significance.

Paraplegics with a lesion below T10 suffered significantly less from constipation $(P=0.002)$.

\section{Use of medication}

Anticholinergics (oxybutynine chloride, oxyfencyclimine hydrochloride, propanthelinebromide) and muscle relaxants (dantrolenum, baclofenum) were used by 32 and 30 patients, respectively. Two patients took antidepressants, three alpha-blocking agents, eight benzodiazepines and three anti-epileptics. In Figure 2 the relationship between constipation and the use of these medications is shown. Statistical analysis was only done for anticholinergics and muscle relaxants, because the other drugs were seldom used. Anticholinergic drug intake was found to be significantly related to constipation $(P=0.03)$. The use of muscle relaxants was higher in Group $C$, but this was not statistically significant. According to our criteria all patients taking laxatives more than once a week (27) belonged to group C. Supplementary dietary fibre was used by nine patients. Thirteen patients were taking a bladder antiseptic.

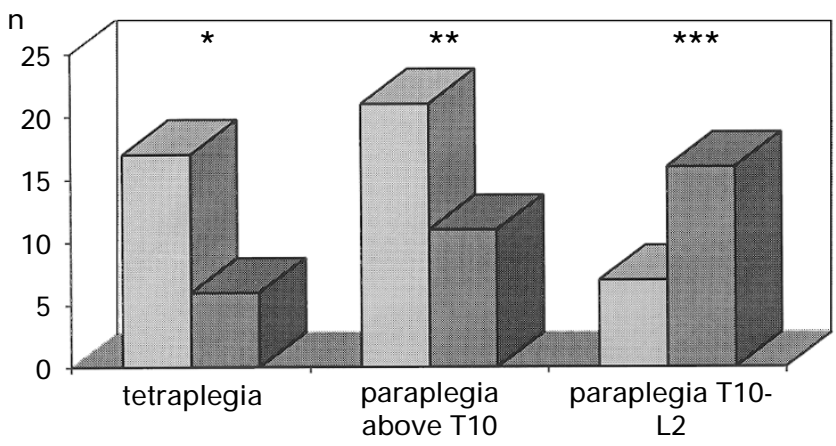

Figure 1 Effect of the level of injury on the prevalence of constipation $(n=78) \square$ Group $\quad \mathrm{C} \square$ Group NC; $* P=0.061, * * P=0.237, * * * P=0.002$ 


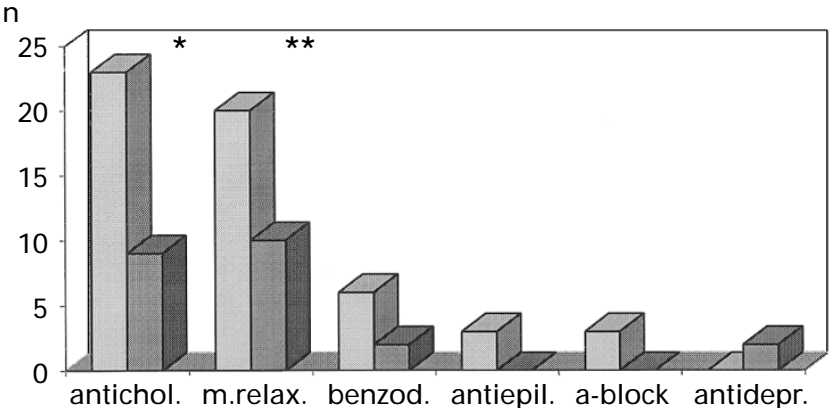

Figure 2 Effect of medication on the prevalence of constipation $(n=78) \square$ Group $\quad \mathrm{C} \square$ Group NC; $* P<0.05, * * P=$ NS. (antichol. $=$ anticholinergics, m.relax. $=$ muscle relaxants, benzod. $=$ benzodiazepines, antiepil. $=$ antiepileptics, a-block $=$ alfa-blockers, antidepr. $=$ antidepressants)

\section{Haemorrhoidal bleeding}

Haemorrhoidal bleeding was mentioned by 17 patients (10 in group $\mathrm{C}$ and seven in group $\mathrm{NC}, P=\mathrm{NS}$ ).

\section{Stool consistency}

The patients were asked to describe stool consistency as it usually presented: very hard, hard, normal or soft. Very hard stools were mentioned by four patients from group $\mathrm{C}$ and none from group NC, hard stools by 19 and 11 patients, normal stools by 19 and 20 patients, and soft stools by three and two patients, respectively. No statistical differences were found between the groups. This analysis confirmed that stool consistency is a very subjective parameter which does not allow us to classify a patient as constipated or not.

\section{Faecal incontinence}

Thirty-seven patients had never experienced faecal incontinence (23 in group C, 14 in group NC). Occasional faecal incontinence was mentioned by 38 patients (19 in group C, 19 in group NC) and daily faecal incontinence by three patients, all belonging to group $\mathrm{C}$.

There was no statistically significant relationship between the presence of constipation and faecal incontinence.

\section{Abdominal pain}

Twenty-four patients had regular abdominal pain. In 20 of them the pain disappeared following a bowel movement. Although abdominal pain was more frequent in group $\mathrm{C}$ (17 patients vs seven patients in group NC), statistical analysis revealed no significant difference. Abdominal distension was mentioned by 25 patients, of whom 19 belonged to group C. Underlying disorders of the gastrointestinal tract were excluded on clinical grounds (no anal blood loss, fever or unexpected weight loss, etc.).

\section{Sensation of defaecation}

In 51 patients some signal was present alerting them to imminent defaecation. Different patterns were reported: rectal distension (seven patients), abdominal pain or pressure (15 patients), passage of air (three patients) mild signs of autonomic dysreflexia (headache, sweating-17 patients) and unspecified sensation (nine patients). The prevalence of constipation was not different in patients with or without these warning signals.

\section{Discussion}

A questionnaire about bowel habits disclosed a $58 \%$ prevalence of constipation in patients with a chronic and complete SCI. Constipation is an ill-defined condition for which criteria have been established in an able-bodied population. ${ }^{7}$ For the purpose of the present study, we only used the criteria of frequency and use of aids, eg laxatives, enema and digital evacuation. Stone et al reported only a $20 \%$ prevalence of 'difficulty with bowel evacuation' in SCI. ${ }^{1}$ Their definition of difficult bowel evacuation (painful defaecation, need for manual evacuation more than once a week or more than $1 \mathrm{~h}$ of bowel care per day) is responsible for this rather low prevalence. We felt that stool frequency was more important in evaluating bowel function and thus obtained a markedly higher prevalence of constipation. Manual evacuation of faeces was needed by only 15 of the 78 patients, not comprising any cauda equina lesions, and equally distributed in tetra- and paraplegics. Anal stimulation was regularly used by 64 or $82 \%$ of our patients. This latter type of 'assisted defaecation' was not considered a manifestation of constipation if stool frequency was higher than twice a week and supplementary laxatives or enemas were not used.

Stone et al found no 'difficulty with evacuation' in patients with injuries of less than 4 years' duration. ${ }^{1}$ We did not find this time-relationship. Also in contrast with the mentioned study, we found constipation to be related to the level of injury. Patients with paraplegia below T10 and above L2, excluding conus and cauda lesions, were significantly less prone to constipation than patients with a higher level of injury $(30 \%$ vs $69 \%, P=0.002)$. In tetraplegics we observed the highest frequency of constipation $(74 \%)$. There is some evidence that patients with low paraplegia have shorter colonic transit times than patients with a higher lesion which would explain this finding (unpublished personal data). Besides the influence of colonic transit time we also have to take into consideration that patients with low paraplegia are able to defaecate in the sitting position and have partially intact abdominal muscles necessary to expel the faeces. Tetraplegic patients on the other hand often defaecate in bed and cannot use their abdominal muscles at all.

The use of anticholinergics was clearly related to the presence of constipation. These drugs may cause 
constipation by prolonging gastric emptying and orocaecal transit time; no data are available about their effect on colonic transit time. ${ }^{8}$ No relationship between the use of muscle relaxants and constipation was found.

Haemorrhoidal bleeding was mentioned by 17 $(21 \%)$ patients, without any relationship however to constipation. This is somewhat higher than the prevalence of haemorrhoidal bleeding in the general population $(4.4 \%$ in the United States, $13 \%$ in Belgium). ${ }^{9,10}$ None of these patients sought medical attention for this problem in the past. Analogous to findings in an able-bodied population, haemorrhoidal bleeding was not related to constipation. ${ }^{9}$

Faecal incontinence was completely absent in almost half of the patients and rarely present in the other half. No relationship was found with constipation, suggesting that constipation does not protect from incontinence. It has been shown that basal pressure at the anal sphincter and anal reflexes remain intact after SCI. ${ }^{11-13}$ These are the most important mechanisms for continence in man. ${ }^{13}$ Three patients who had continuous faecal incontinence, were constipated. In these patients (C4, T5 and T6) we can suspect a mechanism of overflow incontinence due to faecal stasis in the colon.

Regular abdominal pain was present in one third of the patients. The majority of them belonged to group $\mathrm{C}$ and also had abdominal distension. Sixty-two percent of the patients with abdominal pain meet the requirements for Irritable Bowel Syndrome. ${ }^{7}$ We are not aware of any studies that have investigated the presence of IBS in an SCI population and further studies are necessary to confirm this finding.

Although the patients studied had a clinically complete SCI, seven of them had rectal awareness of distension preceding defaecation. Afferent information from the rectal wall is projected to the sacral cord via parasympathetic sacral nerves. ${ }^{14}$ We can speculate that in these seven patients the sacral cord is partially intact and permits afferent information from the lower gastrointestinal tract to reach the central nervous system. This phenomenon of sacral sparing has been well recognized earlier in SCI. ${ }^{15}$ It did not make patients less prone to constipation in our study. The same was true for abdominal pain or autonomic dysreflexia preceeding defaecation: it was as frequent in group $\mathrm{C}$ as in group $\mathrm{NC}$.

\section{Acknowledgements}

We are grateful to $\mathrm{Mr} \mathrm{C}$ Beke (Department of Gastroenterology, University Hospital Ghent) for his help analyzing computer data.

\section{References}

1 Stone J, Nino-Murcia M, Wolfe V, Perkash I. Chronic gastrointestinal problems in spinal cord injury patients: a prospective analysis. Am J Gastroenterol 1990; 85: 1114-1119.

2 Gore R, Mintzer R, Calenoff L. Gastrointestinal complications of spinal cord injury. Spine 1981; 6: $538-544$.

3 Devroede $\mathrm{G}$ et al. Traumatic constipation. Gastroenterology 1979; 77: $1258-1267$.

4 Johanson J, Sonnenberg A, Koch T, McCarty D. Association of constipation with neurologic diseases. Dig Dis Sci 1992; 37: 179_186.

5 McGuire T, Nanda Kumar V. Autonomic dysreflexia in the spinal cord-injured. Postgrad Med 1986; 80: 81 - 89 .

6 Ditunno J, Young W, Donovan W, Creasey G. The international standards booklet for neurological and functional classifiction of spinal cord injury. Paraplegia 1994; 32: 70-80.

7 Thomspon et al. Functional bowel disease and functional abdominal pain. Gastroenterology Int 1992; 5: 75-91.

8 Borody et al. Effects of morphine and atroprine on motility and transit in the human ileium. Gastroenterology 1985; 89: 562-570.

9 Johansson J, Sonnenberg A. The prevalence of hemorrhoids and chronic constipation. An epidemiologic study. Gastroenterology 1990; 98: $380-386$.

10 Belgian Epidemiological Study on Venous Troubles, 1996.

11 Beuret-Blanquart et al. Colonic transit time and anorectal manometric anomalies in 19 patients with complete transection of the spinal cord. J Auton Nerv Syst 1990; 30: 199-208.

12 MacDonagh et al. Anorectal function in patients with complete supraconal spinal cord lesions. Gut 1992; 33: 1532-1538.

13 Sun W et al. Anorectal function in patients with complete spinal cord transection before and after sacral posterior rhizotomy. Gastroenterology 1995; 108: 990 - 998 .

14 Gonella J, Bouvier M, Blanquet F. Extrinsic nervous control of motility of small and large intestines and related sphincters. Physiol Rev 1987; 67: $902-961$.

15 Waters R, Adkins R, Yakura J. Definition of complete spinal cord injury. Paraplegia 1991; 29: 573-581. 